\title{
USO DE MICORRIZAS E RIZÓBIO EM CULTIVO CONSORCIADO DE EUCALIPTO E SESBÂNIA. II - ABSORÇÃO E EFICIÊNCIA DE UTILIZAÇÃO DE FÓSFORO E FRAÇÕES FOSFATADAS ${ }^{(1)}$
}

\author{
L. A. RODRIGUES ${ }^{(2)}$, M. A. MARTINS ${ }^{(3)} \&$ M. S. M. B. SALOMÃO(4)
}

\begin{abstract}
RESUMO
Realizou-se um experimento em casa de vegetação com o objetivo de avaliar os efeitos de fungos micorrízicos arbusculares (FMAs) e rizóbio na absorção e eficiência de utilização de $P$ e nas frações fosfatadas em mudas de Eucalyptus grandis, cultivadas em consorciação com Sesbania virgata. Os tratamentos foram: inoculação ou não com FMAs em ambas as espécies de plantas e inoculação ou não com rizóbio na S. virgata, com quatro repetições. Ambas as plantas foram cultivadas em vasos de $6 \mathrm{~L}$ de capacidade, durante 100 dias, quando foram colhidas. A inoculação com FMAs ou FMAs + rizóbio aumentou o conteúdo de $P$ no eucalipto, enquanto a inoculação com rizóbio, FMAs ou FMAs + rizóbio aumentou a eficiência de utilização de $P$. Nas frações de $P$, avaliadas nas fol has de eucalipto, observou-se aumento do fósforo total solúvel em ácido (PST) nos tratamentos com inoculação de rizóbio ou F MAs + rizobio. Nos tratamentos com inoculação com rizóbio, FMAs, FMAstrizóbio ou sem inoculação, observou-se que 81, 32, 91 e 68\%, respectivamente, do PST foram encontrados como fósforo orgânico (Po). E m uma avaliação conjunta das frações fosfatadas e do conteúdo de $P$ na parte aérea do eucalipto, o que aparentemente influenciou o aumento do PST e do Po não foi o conteúdo interno de P na planta, mas, sim, a inoculação do rizóbio na sesbânia.
\end{abstract}

Termos de indexação: micorrizas arbusculares, leguminosa, associação simbiótica, fixação biológica do nitrogênio.

\footnotetext{
(1) Parte da Tese de Doutorado do primeiro autor, apresentada à Universidade Estadual do Norte Fluminense - UENF. Recebido para publicação em dezembro de 2001 e aprovado em maio de 2003.

(2) Pesquisadora da Universidade Estadual do Norte Fluminense- UENF/CCTA. Av. Alberto Lamego 2000, Campos dos Goytacazes (RJ ). CEP 28013-600. Bolsista da CAPES. E-mail: Iua@uenf.br

(3) Professor Associado, UENF/CCTA. Bolsista do CNPq. E-mail: marco@uenf.br

(4) Aluno do Curso de Agronomia, UENF. Bolsista de Iniciação Cientifica.
} 


\title{
SUMMARY: USE OF MYCORRHIZAS AND RHIZOBIUM IN INTERCROPPING SYSTEM OF EUCALYPTUS AND SESBANIA. II - PHOSPHORUS UPTAKE AND EFFICIENCY OF USE AND PHOSPHATE- FRACTIONS
}

\begin{abstract}
The effects of arbuscular mycorrhizal fungi (AMF) and Rhizobium on P uptake and efficiency of use, as well as on theP fractions of Eucalyptus grandis grown in an intercropping system with Sesbania virgata wereeval uated under greenhouseconditions. Thetreatments were: with or without inoculation with AMF of both plant species and with or without inoculation with Rhizobium of S. virgata plants only, in four replications. The two plant species were grown together in pots with a volume of $6 \mathrm{~L}$ for 100 days. Inoculations with AMF or with AMF + Rhizobium increased the $\mathrm{P}$ content in Eucalyptus plants, while inoculations with Rhizobium, AMF, or with AMF + Rhizobium increased the P utilization efficiency. An increase of total acid-soluble P (PTS) in the treatments inoculated with Rhizobium or AMF + Rhizobium was observed in theP fractions of theanalyzed E ucalyptus plant leaves. In the inoculation treatments with Rhizobium, AMF, and AMF + Rhizobium, and the control (uninoculated) $81,32,91$, and $68 \%$, respectively, of thePST in the leaves of Eucalyptus plants were found in organic-P (Po) form. An evaluation of both the P fractions and P contents of Eucalyptus shoots suggests that the increase of PST and organic-P was caused by theinoculation of Rhizobium in sesbania plants, and not by theinternal P plant content.
\end{abstract}

Index terms: arbuscular mycorrhiza, legumes, symbiotic association, biological nitrogen fixation.

\section{NTRODUÇÃO}

Os fungos micorrízicos arbusculares (FMAs) associam-se às raízes da maioria das espécies de plantas esão capazes de absorver nutrientes do solo que são transferidos para as raízes do hospedeiro. Muitos trabal hos mostram as respostas positivas das plantas, quando inoculadas com FMAs, principalmente em relação à nutrição de $P$ (Hayman,1983; Siqueira \& Paula, 1986; Taiz \& Zeiger, 1991; Marschner \& Dell, 1994; Martins \& Read 1996).

O processo de absorção de $\mathrm{P}$ é similar na hifa micorrízica e na raiz e depende da extensão da hifa externa, bem como do requerimento de $\mathrm{P}$ da planta hospedeira. O fosfato é absorvido pelas hifas micorrízicas e estocado nos tecidos do fungo como grânulos de polifosfatos inorgânicos, sendo remobilizado quando a absorção do $\mathrm{P}$ do solo for insuficiente para o crescimento da planta (Grove et al., 1996).

De acordo com Bieleski (1973), o incremento na absorção do fosfato por plantas micorrizadas pode ser atribuído à maior taxa de conversão de $\mathrm{P}$ inorgânico ( $\mathrm{Pi}$ ) em P-orgânico (Po). Entretanto, as difer enças nas concentrações de P-total observadas nas plantas podem resultar deacúmulos diferenciais de P-inorgânico nas plantas e não de P-orgânico (Martinez et al., 1993a; Martinez et al., 1993b). Segundo Ozanne (1980), o P-inorgânico pode ser estocado nos vacúol os e liberado para o citossol da célula, conformea demanda da planta. Desta forma, a planta teria um tampão interno ou um fator capacidade interno de P (Martinez et al., 1993a, Martinez et al., 2002), à semel hança do que ocorre no solo (Ozanne, 1980).

No cultivo de plantas consorciadas com leguminosas, a transferência de nutrientes da decomposição de nódulos eraízes ou da serapilheira da leguminosa, por meio das hifas micorrízicas, pode mel horar a nutrição dessas plantas, principal mente em relação ao N e P (Newman, 1988; Frey \& Schuepp, 1992; Martensson et al., 1998). Noentanto, o transporte desses nutrientes das hifas é bidirecional (Sen, 2000), podendo ocorrer competição entre as plantas interconectadas em uma mesma rede micelial (N ewman, 1988).

Estudos sobre a absorção e distribuição interna do $P$ e das frações fosfatadas podem embasar as respostas sobre a transferência do $P$ do fungo para a planta e são importantes para o entendimento dos processos de adaptação das plantas à deficiência de $\mathrm{P}$, quando duas espécies estão sendo cultivadas em consórcio.

O objetivo deste trabal ho foi avaliar o acúmulo e a eficiência de utilização deP e as frações fosfatadas por plantas de eucalipto (E . grandis), inoculadas ou não com FMAs, cultivadas em consorciação com plantas de sesbânia, inoculadas ou não com rizóbio e, ou, FMAs. 


\section{MATERIAL E MÉTODOS}

O experimento foi realizado em casa de vegetação no "Campus" da Universidade Estadual do Norte Fluminense, em Campos dos Goytacazes, com cultivo em vasos de Eucalyptus grandis consorciado com Sesbania virgata. Utilizou-se o delineamento experimental inteiramente casualizado com 4 tratamentos, sendo: inoculação ou não do eucalipto e da sesbânia com FMAs e inoculação ou não da sesbânia com rizóbio. Cada tratamento foi realizado com quatro repetições.

A obtenção emultiplicação dos inóculos dos F MAs e do rizóbio, a produção de plântulas inoculadas e não inoculadas, o preparo dos vasos e do substrato, o cultivo das plântulas e a col heita do experimento foram realizados de acordo com os procedimentos descritos em Rodrigues et al. (2003).

\section{Análise do fósforo e frações fosfatadas}

Na ocasião da col heita, foram col etadas amostras de aproximadamente $2 \mathrm{~g}$ de fol has, completamente expandidas de eucal ipto do terço mediano dos gal hos. As amostras foram pesadas, enxaguadas em água deionizada, acondicionadas imersas em ácido perclórico $0,2 \mathrm{~mol} \mathrm{~L}^{-1}$ e congeladas para posterior análise de fósforo total solúvel em ácido (PST) e fósforo inorgânico solúvel (Pi).

Para isto, as amostras de fol has congeladas em ácido perclórico $0,2 \mathrm{~mol} \mathrm{~L}^{-1}$ foram maceradas e centrifugadas a $5.000 \mathrm{~g}$, segundo método descrito por Smillie \& Krotkov (1960) e modificado por Martinez (1992). A fração inorgânica (Pi) foi determinada no sobrenadante do material centrifugado. A fração do $\mathrm{P}$ total solúvel em ácido (PST) foi determinada após digestão nitroperclórica do sobrenadante do material centrifugado. As determinações do PST e $\mathrm{Pi}$ foram realizadas pelo método do azul de molibdênio, tendo ácido ascórbico como agente redutor (Braga \& Defeli po, 1974). O fósforo orgânico (Po) foi calculado pela diferença entre o PST e o Pi. Calculou-se também o percentual relativo de Pi e Po em relação ao PST.

As raízes da sesbânia (após a retirada dos nódulos) e do eucalipto e a parte aérea das duas espécies foram secas, separadamente, em estufa de circulação forçada dear a $65^{\circ} \mathrm{C}$ por $72 \mathrm{~h}$. O material foi moído em moinho tipo Wiley, com peneira de 20 malhas por polegada. Amostras de $200 \mathrm{mg}$ do material vegetal seco e moído (parte aérea e raízes) foram submeti das à digestão nítrico-perclórica e, por col orimetria, foram determinadas as concentrações de $P$ nas amostras pelo método do azul de molibdênio, tendo ácido ascórbico como agente redutor (Braga \& Defelipo, 1974). A eficiência de utilização de $P$ (EFU-P) nas plantas de eucalipto e sesbânia foi calculada pela seguinte fórmula: EFU $=\left[(\text { matéria seca })^{2} /(\right.$ conteúdo do nutriente) $]$ (Sidiqui $\&$ Glass, 1981).
Os dados foram submetidos à análise de variância e teste de média, por Tukey a $5 \%$, usando-se o programa SAEG (Sistema de Análise Estatística e Genética) (Euclydes, 1983).

\section{RESULTADOS E DISCUSSÃO}

As plantas de eucalipto dos tratamentos sem inoculação com os microrganismos (tratamentocontrole) e do tratamento com inoculação apenas do rizóbio apresentaram, inicial mente, um amarel ecimento nas fol has mais vel has, que, posteriormente, atingiram, também, as fol has jovens. Aos cem dias deidade(na ocasião da col heita), as fol has deeucalipto desses tratamentos apresentavam coloração amarelo-avermelhada, com pontos arroxeados no limbo foliar emenor tamanho em relação às plantas inoculadas com FMAs ou FMAs + rizóbio. $\mathrm{Na}$ sesbânia, não foram observados quaisquer sintomas visuais de deficiência mineral na parte aérea em nenhum dos tratamentos.

A inoculação com FMAs + rizóbio proporcionou maior teor de $\mathrm{P}$ na parte aérea e na planta toda do eucalipto, quando comparada somente com a inocul ação com o rizóbio ou somente com a inoculação com os FMAs. Além disto, tanto na planta toda quanto nas raízes, a inoculação com os FMAs promoveu o aumento no teor de $\mathrm{P}$ em relação ao tratamentocontrole (sem inoculação com os microrganismos).

Na sesbânia, a inoculação com o rizóbio aumentou o teor de $\mathrm{P}$ nas raízes, mesmo quando as plantas não estavam inoculadas com os FMAs (Quadro 1). J á o efeito dos FMAs em proporcionar aumento no teor deP ocorreu na parteaérea etotal, mas somente sem a inoculação do rizóbio (Quadro 1).

Os teores de $\mathrm{P}$ na parte aérea do eucalipto do tratamento-controle e do tratamento com rizóbio $\left(0,76\right.$ e $0,8 \mathrm{~g} \mathrm{~kg}^{-1}$, respectivamente) não atingiram os níveis adequados para as plantas. Os teores de $P$ considerados adequados para mudas de $\mathrm{E}$. grandis estão na faixa entre 0,9 e $2,6 \mathrm{~g} \mathrm{~kg}^{-1}$ (Barros et al., 1990; Dell, 1996; J udd et al., 1996; Malavolta et al., 1997).

Este resultado justificaria o sintoma visual de deficiência nutricional observadonesses tratamentos. Entretanto, os teores de $\mathrm{N}$ também se encontravam abaixo daqueles considerados adequados para o eucal ipto em todos os tratamentos (Rodrigues et al., 2002). Os sintomas de deficiência de $\mathrm{N}$ em plantas de eucalipto, segundo Dell (1996), iniciam com clorose nas folhas velhas, tendo em vista o decréscimo da formação de clor ofila, acompanhada de menor tamanho das folhas. Deacordo com Novais et al. (1990), na deficiência de N, a cloroseéuniforme, com amarelecimento das fol has seguido da queda das mesmas. J á os sintomas de deficiência de P são expressos por pontos e manchas escuro-arroxeadas sobre um limbo foliar verde intenso. 
Quadro 1. Teores e eficiência de utilização de P nas plantas de eucalipto e sesbânia, inoculadas ou não com rizóbio (riz) e fungos micorrízicos arbusculares (FMAs), cultivadas em consorciação

\begin{tabular}{|c|c|c|c|c|c|c|c|}
\hline \multirow{2}{*}{ Espécie } & \multirow{2}{*}{ Tratamento } & \multicolumn{2}{|c|}{ Parte aérea } & \multicolumn{2}{|c|}{ Raiz } & \multicolumn{2}{|c|}{ Total } \\
\hline & & Sem riz & Com riz & Sem riz & Com riz & Sem riz & Com riz \\
\hline & & \multicolumn{6}{|c|}{ Fósforo, $\mathrm{g} \mathrm{kg}^{-1}$} \\
\hline Eucalipto & $\begin{array}{l}\text { Sem FMAs } \\
\text { Com FMAs }\end{array}$ & $\begin{array}{l}0,76 \mathrm{aA} \\
1,20 \mathrm{bA}\end{array}$ & $\begin{array}{l}0,84 \mathrm{aB} \\
1,78 \mathrm{aA}\end{array}$ & $\begin{array}{l}1,60 \mathrm{aB} \\
1,75 \mathrm{aA}\end{array}$ & $\begin{array}{l}1,50 \mathrm{aA} \\
1,65 \mathrm{aA}\end{array}$ & $\begin{array}{l}1,18 \mathrm{aB} \\
1,47 \mathrm{bA}\end{array}$ & $\begin{array}{l}1,24 \mathrm{aB} \\
1,65 \mathrm{aA}\end{array}$ \\
\hline \multirow[t]{2}{*}{ Sesbânia } & $\begin{array}{l}\text { Sem FMAs } \\
\text { Com FMAs }\end{array}$ & $\begin{array}{l}1,34 \mathrm{aB} \\
2,84 \mathrm{aA}\end{array}$ & $\begin{array}{l}1,50 \mathrm{aA} \\
2,46 \mathrm{aA}\end{array}$ & $\begin{array}{l}0,67 \mathrm{bA} \\
0,59 \mathrm{aA}\end{array}$ & $\begin{array}{l}1,44 \mathrm{aA} \\
1,00 \mathrm{aA}\end{array}$ & $\begin{array}{l}1,00 \mathrm{aB} \\
1,71 \mathrm{aA}\end{array}$ & $\begin{array}{l}1,47 \mathrm{aA} \\
1,73 \mathrm{aA}\end{array}$ \\
\hline & & \multicolumn{6}{|c|}{ Fósforo, g vaso ${ }^{1}$} \\
\hline Eucalipto & $\begin{array}{l}\text { Sem FMAs } \\
\text { Com FMAs }\end{array}$ & $\begin{array}{l}3,51 \mathrm{aB} \\
8,68 \mathrm{aA}\end{array}$ & $\begin{array}{l}4,39 a \mathrm{a} \\
9,52 \mathrm{aA}\end{array}$ & $\begin{array}{l}3,03 \mathrm{aB} \\
5,77 \mathrm{aA}\end{array}$ & $\begin{array}{l}3,84 \mathrm{aA} \\
5,18 \mathrm{aA}\end{array}$ & $\begin{array}{r}6,53 \mathrm{aB} \\
14,45 \mathrm{aA}\end{array}$ & $\begin{array}{r}8,21 \mathrm{aB} \\
14,69 \mathrm{aA}\end{array}$ \\
\hline Sesbânia & $\begin{array}{l}\text { Sem FMAs } \\
\text { Com FMAs }\end{array}$ & $\begin{array}{l}13,87 \mathrm{bA} \\
36,63 \mathrm{aA}\end{array}$ & $\begin{array}{l}21,53 \mathrm{aA} \\
36,75 \mathrm{aA}\end{array}$ & $\begin{array}{l}3,22 \mathrm{bA} \\
3,28 \mathrm{aA}\end{array}$ & $\begin{array}{l}7,95 \mathrm{aA} \\
6,31 \mathrm{aA}\end{array}$ & $\begin{array}{l}17,09 \mathrm{aB} \\
39,91 \mathrm{aA}\end{array}$ & $\begin{array}{l}29,48 a A \\
43,09 a A\end{array}$ \\
\hline & & \multicolumn{6}{|c|}{ Eficiência de utilização de $P, \mathrm{mg}^{2} \mathrm{mg}^{-1}$} \\
\hline Eucalipto & $\begin{array}{l}\text { Sem FMAs } \\
\text { Com FMAs }\end{array}$ & - & - & $\begin{array}{l}- \\
-\end{array}$ & $\begin{array}{l}- \\
-\end{array}$ & $\begin{array}{l}1.610,48 \mathrm{bB} \\
4.057,07 \mathrm{aA}\end{array}$ & $\begin{array}{l}3.650,88 \mathrm{aA} \\
4.073,80 \mathrm{aA}\end{array}$ \\
\hline Sesbânia & $\begin{array}{l}\text { Sem FMAs } \\
\text { Com FMAs }\end{array}$ & - & - & - & - & $\begin{array}{l}7.099,80 \mathrm{aA} \\
4.303,50 \mathrm{aA}\end{array}$ & $\begin{array}{l}6.544,30 \mathrm{aA} \\
4.717,30 \mathrm{aA}\end{array}$ \\
\hline
\end{tabular}

Para uma mesma espécie e mesma parte da planta, as médias seguidas pela mesma letra minúscula, na linha, ou maiúscula, na coluna, não diferem entre si (teste de Tukey a $5 \%$ ).

Assim, o sintoma visual de deficiência nutricional observado nas plantas do tratamento-controleecom rizóbio pode ser devido não somenteà deficiência de $P$, mas também a interações fisiológicas promovidas pelo baixo teor de $\mathrm{N}$, uma vez que os demais nutrientes foram adicionados em quantidades adequadas para a nutrição do eucalipto, segundo as indicações sugeridas por Lopes \& Guimarães (1989) e Barros et al. (1990).

Apesar de não terem sido observados sintomas visíveis de deficiência mineral na parte aérea das plantas de sesbânia, o aumento nos teores de $\mathrm{P}$ nas plantas inoculadas com FMAs e FMAstrizóbio (Quadro 1) foi acompanhado do aumento no peso da matéria seca na parte aérea, indicando que, provavelmente, este nutriente poderia estar limitando o crescimento das plantas nos tratamentocontrole einoculado somente com rizóbio (Rodrigues et al., 2003).

$\mathrm{Na}$ maioria das culturas, a relação $\mathrm{P} / \mathrm{N}$, necessária para haver um balanço adequado entre estes nutrientes na planta, éde 1:10 (Ozanne, 1980). A relação P/N nas plantas de eucalipto foi de 1:7, 1:7, 1:6 e 1:7, para o tratamento-controle, com rizóbio, com FMAs e com FMAs + rizóbio, respectivamente. A baixa relação $\mathrm{P} / \mathrm{N}$ deve-se provavel menteaoteor de $\mathrm{N}$ abaixo do adequado para as plantas de eucalipto em todos os tratamentos.
Nas plantas desesbânia, a relação $\mathrm{P} / \mathrm{N}$ foi de $1: 15$, 1:17, 1:10 e 1:12 para o tratamento-controle, com rizóbio, com FMAs e com FMAs + rizóbio, respectivamente. Nos tratamentos com FMAs e FMAs + rizóbio, o teor de $\mathrm{P}$ estando mais adequado consegue manter uma relação $\mathrm{P} / \mathrm{N}$ mais próxima ao que Ozanne (1980) considerou como sendo mais indicado para as plantas.

Na parte aérea, nas raízes e na planta toda do eucalipto, a inoculação com o rizóbio não proporcionou alterações significativas no conteúdo de $P$, independentemente de as plantas estarem ou não inoculadas com os FMAs (Quadro 1). Noentanto, a inoculação com os FMAs aumentou o conteúdo de $\mathrm{P}$ na parte aérea e total, independentemente de as plantas estarem ou não inoculadas com o rizóbio, e nas raízes, mas somente em relação ao controle.

Observou-se aumento no conteúdo de $\mathrm{P}$ na parte aérea e nas raízes da sesbânia com a inoculação com o rizóbio, bem como aumento no conteúdo de $\mathrm{P}$ na planta toda com a inoculação com os FMAs.

A eficiência de utilização de P (EFU-P) nas plantas de eucalipto (total) aumentou com a inoculação do rizóbio, dos FMAs e FMAs + rizóbio (Quadro 2). Nos demais tratamentos ena sesbânia, não foram verificadas diferenças significativas na EFU-P. Os incrementos na EFU-P nas plantas de 
eucalipto, comparados aos do tratamento-controle, foram de 126, 152 e $153 \%$, para os tratamentos com rizóbio, FMAs ou FMAs + rizóbio, respectivamente, indicando a importância não somente da micorriza, mas também do rizóbio em relação à nutrição de $P$, no cultivo consorciado.

Em relação às frações fosfatadas nas fol has de eucalipto, observou-se que a inoculação do rizóbio promoveu o aumento dofósforototal solúvel em ácido (PST) e do fósforo orgânico (Po) (Quadro 2), estando as plantas inoculadas ou não com os FMAs. A inoculação dos FMAs + rizóbio proporcionou decréscimo no fósforo inorgânico $(\mathrm{Pi})$ em relação à inoculação somente com os F MAs e somente com o rizóbio. A inoculação com os FMAs proporcionou aumento no Pi e decréscimo no Po (Quadro 2).

F oram calculados os percentuais de $\mathrm{Pi}$ e Po nas fol has das plantas de eucalipto em relação ao PST (Quadro 2), para indicar o percentual de acúmulo do $\mathrm{P}$ vacuolar $(\mathrm{Pi})$ e o percentual de $\mathrm{P}$ metabólico (Po). I ndependentemente da inoculação ou não com os FMAs, a inoculação do rizóbio proporcionou decréscimo no percentual de $\mathrm{Pi}$ e conseqüente acréscimo no percentual de Po, enquanto a inoculação com os FMAs aumentou o percentual de $\mathrm{Pi}$ e diminuiu o percentual de Po. A inoculação com os FMAs + rizóbio resultou em decréscimo no percentual de Pi e acréscimo no percentual de Po, quando se compararam plantas inoculadas somente com rizóbio ou somente com os FMAs.

De acordo com Ozanne (1980), as diferenças nas concentrações de $\mathrm{P}$ solúvel total são resultantes de acúmulos diferenciais dePi nas plantas, enquanto as concentrações de Po variam pouco, independentemente da disponibilidade de $\mathrm{P}$ no solo.

No entanto, no presente trabalho, as plantas de eucalipto do tratamento-controle ou com rizóbio apresentaram sintomas de deficiências nutricionais visíveis nas fol has, acompanhados de teores de $\mathrm{N}$ (Rodrigues et al., 2003) e de P abaixo dos níveis adequados para mudas de eucalipto (Barros et al., 1990; Dell, 1996; J udd et al., 1996; Malavolta et al., 1997). Neste caso, as plantas, provavelmente, não estavam acumulando o P (como $\mathrm{Pi}$ ) e estariam preferencial mente utilizando o P para seu metabolismo.

Esse fato pode ser confirmado pelo resultado na percentagem de P em relação ao PST (Quadro 2), quando, para o tratamento-controle e com rizóbio, observou-se que 67,5 e $81,2 \%$ do PST foram encontrados na forma de Po e somente os 32,5 e $18,8 \%$, restantes, como $\mathrm{Pi}$, respectivamente, confirmando, portanto, os resultados de Ozanne (1980) e de Fernandes et al. (2000), que observaram maiores concentrações de Po em relação ao Pi em plantas de feijão, quando submetidas a baixas doses de $P$. Respostas semelhantes também foram relatadas por Martinez et al. (1993a) e Martinez et al. (2002), em plantas de soja.

A percentagem relativa de $\mathrm{Pi}$ nas plantas inoculadas somente com FMAs foi de $68,5 \%$ (Quadro 2), indicando que as plantas já estariam acumulando o $\mathrm{P}$ como reserva vacuolar (Pi). Neste tratamento, as plantas de eucalipto apresentaram teor es adequados deP enão apresentaram sintomas visuais de deficiência nutricional. Além disto, as respostas observadas no conteúdo de $\mathrm{P}$ nas raízes do eucal ipto, comparadas ao resultado da parteaérea (Quadro 1), indicam que, nas raízes, as plantas mantiveram um nível de $\mathrm{P}$ mais estável. Desta forma, o P absorvido em excesso pelas plantas no tratamento com F MAs estaria sendo acumulado na parte aérea.

Furtini Neto et al. (1998) observaram que plantas de E. grandis em sol ução nutritiva, sem omissão de

Quadro 2. Fósforo total solúvel em ácido (PST), fósforo inorgânico (Pi) e fósforo orgânico (Po) nas folhas de plantas de eucalipto, cultivadas em consorciação com sesbânia, inoculadas ou não com rizóbio (riz) e fungos micorrízicos arbusculares (F MAs)

\begin{tabular}{|c|c|c|c|c|c|c|}
\hline \multirow{2}{*}{ Tratamento } & \multicolumn{2}{|c|}{ PST } & \multicolumn{2}{|c|}{$\mathbf{P i}$} & \multicolumn{2}{|c|}{ Po } \\
\hline & Sem riz & Com riz & Sem riz & Com riz & Sem riz & Com riz \\
\hline & \multicolumn{6}{|c|}{ Teor, $\mathrm{g} \mathrm{kg}^{-1}$} \\
\hline Sem FMAs & $46,95 \mathrm{bB}$ & $113,71 \mathrm{aA}$ & $15,25 \mathrm{aB}$ & 20,11 aA & $31,70 \mathrm{bA}$ & $93,60 \mathrm{aA}$ \\
\hline \multirow[t]{2}{*}{ Com FMAs } & 47,52 bA & $92,70 \mathrm{aA}$ & $25,22 \mathrm{aA}$ & $8,30 \mathrm{bB}$ & $22,30 \mathrm{bB}$ & $84,40 \mathrm{aA}$ \\
\hline & \multicolumn{6}{|c|}{ Partici pação relativa, \% } \\
\hline Sem FMAs & - & - & $32,50 \mathrm{aB}$ & $18,80 \mathrm{bA}$ & $67,50 \mathrm{bA}$ & $81,20 a B$ \\
\hline Com FMAs & - & - & $68,50 \mathrm{aA}$ & $9,00 \mathrm{bB}$ & $31,50 \mathrm{bB}$ & $91,00 \mathrm{aA}$ \\
\hline
\end{tabular}

Para uma mesma fração de $\mathrm{P}$, as médias seguidas pela mesma letra minúscula, na linha, ou maiúscula, na colunam, não diferem entre si (teste de Tukey a $5 \%$ ). 
P, apresentaram 46 e $54 \%$ do PST como Pi e Po, respectivamente, em folhas basais. Aos 20 dias de omissão de $P$, ou seja, sob condições de deficiência deste elemento, ocorreram aumento percentual no Poe decréscimo no Pi. Neste caso, 17 e $83 \%$ do PST foram encontrados como $\mathrm{Pi}$ e $\mathrm{Po}$, respectivamente. Os resultados relatados pel os autores no tratamento sem omissão de $\mathrm{P}$ podem ser comparados aos resultados obtidos com a inoculaçao dos FMAs, no presente trabalho, em que as plantas conseguiram adquirir maior conteúdo de $\mathrm{P}$ na matéria seca $\mathrm{e}$ maior percentual relativo de Pi (68,9\%), indicando acúmulo de $P$.

De acordo com Malavolta et al. (1997), plantas deficientes em $\mathrm{P}$ apresentam maior relação $\mathrm{Po} / \mathrm{Pi}$ que plantas sem deficiência de $\mathrm{P}$. As relações $\mathrm{Po} / \mathrm{Pi}$ foram de 2,1, 4,64 e 0,88, para o tratamento-controle, com rizóbio ecom $\mathrm{FMAs}$, respectivamente. As respostas observadas na relação $\mathrm{Po} / \mathrm{Pi}$, são justificadas pelos baixos teores de $\mathrm{P}$ observados no tratamentocontrole e com rizóbio e teores mais elevados no tratamento com FMAs.

A inoculação com F MAs + rizóbio nas plantas de eucalipto, no entanto, levou a uma alta relação Po/ $\mathrm{Pi}$ (que foi 10,2), embora as plantas não apresentassem sintomas visuais de deficiência mineral, apresentassem teor adequado de $\mathrm{P}$ na matéria seca e, ainda, conteúdo de $\mathrm{P}$ maior que aqueles observados no tratamento-controle e com rizóbio (Quadro 1). No tratamento com FMAs + rizóbio, $91 \%$ do PST foi encontrado como Po e somente $9 \%$ como Pi (Quadro 2).

Estes resultados contrastam com os citados por Malavolta et al. (1997) e ao discutido por Ozanne (1980), Martinez et al. (1993a), Furtini Neto et al. (1998) eF ernandez et al. (2000). U ma hipóteseseria a maior conversão de $\mathrm{Pi}$ em Po na parte aérea de plantas micorrizadas, para manter baixo o teor de $\mathrm{Pi}$ nas fol has e proporcionar um maior gradiente de Pi entre as raízes (micorrizadas) e a parte aérea e, por conseqüência, aumentar o dreno de fosfato do solo para as hifas micorrízicas (Bieleski, 1973).

É interessante relacionar o resultado observado nas frações fosfatadas com as respostas observadas na aquisição de $\mathrm{N}$ nas plantas de eucalipto (Rodrigues et al., 2003), nos tratamentos com inoculação com FMAs + rizóbio. N estetratamento, as plantas de eucalipto apresentaram maior teor e conteúdo de N (total), que pode ter sido proveniente da adubação do solo, da decomposição de raízes e nódulos da sesbânia ou, ainda, do transporte, via hifa micorrízica, de $\mathrm{N}$ e de compostos orgânicos da sesbânia para o eucalipto. Esta mai or aquisição de $\mathrm{N}$ no eucalipto pode ter levado à formação de compostos orgânicos ou de proteínas na planta, requerendo maior estoque metabólico de P na planta com conseqüente diminuição do $\mathrm{Pi}$ nos tratamentos com F MAs + rizóbio.
O acréscimo no teor de Po nos tratamentos com rizóbio e FMAs + rizóbio, comparado ao dos demais tratamentos, revela que a maior influência no aumento do Po não seria o teor ou o conteúdo interno deP na planta de eucal ipto, como sugerido por vários autores, mas, sim, a presença do rizóbio inoculado na sesbânia consorciada ao eucalipto.

\section{CONCLUSÕES}

1. As inoculações com FMAs ou com FMAs + rizóbio proporcionaram mai or teor e conteúdo de $\mathrm{P}$ nas plantas de eucalipto e de sesbânia, cultivadas em consorciação, e maior eficiência de utilização de $P$ pelas plantas de eucalipto.

2. A inoculação com os microrganismos causou variações nas frações fosfatadas das fol has de eucalipto, tendo a inoculação com rizóbio ou FMAs + rizóbio aumentado os teores de PST e Po.

\section{LITE RATURA CITADA}

BARROS, N.F.; NOVAIS, R.F. \& NEVES, J.C.L. Fertilização e correção do solo para o plantio de eucalipto. In: BARROS, N.F. \& NOVAIS, R.F., eds. Relação solo-eucalipto. Viçosa, Folha de Viçosa, 1990. p 127-186.

BIELESKI, B.L. Phosphate pools, phosphate transport, and phosphate availability. Ann. Rev. Plant Physiol., 24:225252, 1973.

BRAGA, J.M. \& DEFELIPO, B.V. Determinação espectrofotomética do fósforo com extrato de solos e plantas. R. Ceres, 41:73-85, 1974.

DELL, B. Diagnosis of nutrient deficiencies in eucalypts. In: ATTIWILL, P.M. \& ADAMS, M.A. eds. Nutrition of Eucalypts. Australia, 1996. p.417-440.

EUCLYDES, R.F. Sistema para análise estatísticas e genéticas (SAEG). Viçosa, U niversidade Federal de Viçosa, 1983. 74p.

FERNANDEZ, L.A.; FAQUIN, V.; FURTINI NETO, A.E. \& CURI, N. Frações de fósforo e atividade da fosfatase ácida em plantas de feijoeiro cultivadas em solos de várzea. R. Bras. Ci. Solo, 24:561-571, 2000.

FREY, B. \& SCHUEPP, H. Transfer of symbiotically fixed nitrogen from berseen (Trifol ium al exandrinum L.) to maize via vesicular-arbuscular mycorrhizal hyphae. New Phytol., 122:447-454, 1992.

FURTINI NETO, A.E.; BARROS, N.F.; NOVAIS, R.F. \& OLIVEIRA, M.F.G. Frações fosfatadas em mudas de Eucalyptus. R. Bras. Ci. Solo, 22:267-274, 1998.

GROVE, T.S.; THOMSON, B.D. \& MALAJ CZUK, N. Nutritional physiology of eucalypts: uptake, distribution and utilization. In: ATTIWILL, P.M. \& ADAMS, M.A., eds. Nutrition of Eucalypts. Australia, 1996. p.77-108. 
LOPES, A.S. \& GUIMARÃES, P.T.G. Recomendação para o uso de corretivos e fertilizantes em Minas Gerais. 4a. aproximação. Lavras, Comissão de Fertilidade do Solo do Estado de Minas Gerais, 1989. 159p.

HAYMAN, D.S. The Physiology of VA endomycorrhizal symbiosis. Can. J . Bot., 61:944-963, 1983.

J UDD, T.S.; ATTIWILL P.M. \& ADAMS, M.A. Nutrient concentration in Eucalyptus: a synthesis in relation to differences between taxa, sites and components. In: ATTIWILL, P.M. \& ADAMS, M.A., eds. Nutrition of eucalypts. Australia, 1996 p.123-154.

MALAVOLTA, E.; VITTI, G.C. \& OLIVEIRA, S.A. Avaliação de estado nutricional das plantas. 2.ed. Piracicaba, POTAFOS, 1997. 367p.

MARSCHNER, H. \& DELL, B. Nutrient uptake in mycorrhizal symbiosis. Plant Soil, 159:89-102, 1994.

MARTENSSON, A.M.; RYDBERG, I. \& VESTBERG, M. Potential to improve transfer of $\mathrm{N}$ in intercropped systems by optimizing host-endophyte combinations. Plant Soil, 205:57-66, 1998.

MARTINEZ, H.E.P. Cinética da absorção e das frações fosfatadas em sete variedades de soja (Glycinemax, L. Merril). Viçosa, Universidade Federal de Viçosa, 1992. Não paginado. (Relatório de Pós-Doutoramento)

MARTINEZ, H.P.; NOVAIS, R.F.; RODRIGUES, L.A.; SACRAMENTO, L.V.S. \& REIS J UNIOR, R.A. Reactions of three soybean cultivars to interruptions in phosphorus supply. In: FRAGOSO, M.A.C. \& VAN BEUSICHEM, M.L., eds. Optimization of plant nutrition. Dordrecht, 1993a. p.547-554.

MARTINEZ, H.P.; NOVAIS, R.F.; RODRIGUES, L.A. \& SACRAMENTO, L.V.S. Phosphate forms in the plant and their internal buffering in five soybean cultivars. In: COMUNICACIONES - SIMPOSIO IBÉRICO SOBRE NUTRICIÓN MINERAL DE LAS PLANTAS, 9., Zaragoza, 2002. Zaragoza, Institución Fernando el Católico, 2002. p.67-70.
MARTINEZ, H.P.; NOVAIS, R.F.; RODRIGUES, L.A. \& SACRAMENTO, L.V.S. Comportamento de variedades de soja cultivadas em diferentes doses de fósforo: Cinética de absorção de fósforo e ajustes morfológicos da planta. R. Bras. Ci. Solo, 17:231-238, 1993b.

MARTINS, M.A. \& READ, D.J . Therole of the external mycelial network of VA mycorrhizal fungi : II . A study of phosphorus transfer between plants interconnected by a common mycelium. R. Microb., 27:30-35, 1996.

NEWMAN, E.I. Mycorrhizal links betwen plants: Their functioning and ecological significance. Adv. Ecol. Res., 18:243-270, 1988.

NOVAIS, R.F.; BARROS, N.F. \& NEVES, J.C.L. Nutrição mineral de eucalipto. In: BARROS, N.F. \& NOVAIS R.F., eds. Relação solo-eucalipto. Viçosa, Fol ha de Viçosa, 1990. p.25-98.

OZANNE, P.G. Phosphate Nutrition of plants - A general treatise. In: KHASAWNEH, F.E.; SAMPLE, E.C. \& KAMPRATH, E.J., eds. The role of phosphorus in agriculture. Madison, American Society of Agronomy, Crop Science Society of America, Soil Science Society of America, 1980. p.559-585.

RODRIGUES, L.A.; MARTINS, M.A. \& SALOMÃO, M.S. Uso de mi corrizas e rizóbio em cultivos consorciados de eucal ipto e sesbânia. I - Crescimento, absorção e transferência de N entre as plantas. R. Bras. Ci. Solo, 4:583-591, 2003.

SEN, R. Budgesting for the wood-wide web. New Phytol., 145:161-165, 2000. Fórum Commentary.

SIDIQUI, M.Y. \& GLASS, A.D.M. Utilization index: a modified approach to the estimation and comparation of nutrient utilization efficiency in plants. J . Plant Nut., 4:289-302, 1981.

SIQUEIRA, J .O. \& PAULA, M.A. Efeito de micorrizas vesículoarbusculares na nutrição e aproveitamento de fósforo pela soja em sol os do cerrado. R. Bras. Ci. Solo, 10:97-102, 1986.

SMILLIE, R.M. \& KROTKOV, G. The estimation of nucleic acids in somealgaeand higher plants. Can. J . Bot., 38:31-49, 1960.

TAIZ, L. \& ZEIGER, E. Plant physiology. Redwood, The Benjamin Cummings Publishing Company, 1991. 559p. 